\title{
Link between Magnetic and Dielectric Properties in Magnetite
}

\author{
Z. ŠVINDRYCH ${ }^{a, b}$, A. YOUSSEF ${ }^{b}$ AND Z. JANÜ ${ }^{a, *}$ \\ ${ }^{a}$ Institute of Physics of the AS CR, v.v.i, Na Slovance 2, 18221 Prague, Czech Republic \\ ${ }^{b}$ Charles University in Prague, Faculty of Mathematics and Physics, V Holešovičkách 2, 18000 Prague, Czech \\ Republic
}

We have measured temperature dependence of magnetic and dielectric properties in high quality stoichiometric single-crystals of magnetite $\left(\mathrm{Fe}_{3} \mathrm{O}_{4}\right)$. At low temperatures, below the Verwey transition, both ac susceptibility and ac permittivity reveal similar relaxation processes. We discuss these effects in connection with domain structure and variable range hopping mechanism of electrical conductivity.

PACS numbers: 71.30. $+\mathrm{h}, 75.60 .-\mathrm{d}, 75.85 .+\mathrm{t}$

\section{Introduction}

Magnetite - a mixed valence iron oxide, $\mathrm{Fe}_{3} \mathrm{O}_{4}$, with cubic inverse spinel structure at room temperature - is a prototype ferrimagnetic system that still attracts considerable attention mainly due to the presence of the so-called Verwey transition — a structural phase transition accompanied by substantial changes of magnetic and electrical properties [1]. Although Mott's metalinsulator transition theory [2] was well suited to explain the sharp increase of resistivity of magnetite upon cooling through the Verwey temperature $\left(T_{\mathrm{V}} \approx 120 \mathrm{~K}\right)$, the exact nature of the transition is still unresolved and the charge ordering on the Fe ions has not been sufficiently clarified [3-6]. To complicate the matter even further, upon cooling through $T_{\mathrm{V}}$ the structure changes to monoclinic [5] and the monocrystal splits into structural domains which are strongly linked to the instantaneous magnetic domain structure of the sample (i.e. random in weak magnetic fields). The interplay of spin, lattice and electronic degrees of freedom makes this strongly correlated system a potential candidate for novel applications [7].

In this work we focus not on the Verwey transition itself but rather on the magnetic and dielectric behavior of magnetite below $T_{\mathrm{V}}$.

Stoichiometric single crystals of magnetite were grown by floating zone technique [8] and cut to disk shape $5 \mathrm{~mm}$ in diameter and $1 \mathrm{~mm}$ thick, the disk axis corresponds to $\langle 100\rangle$ crystallographic direction. Two silver electrodes (4 $\mathrm{mm}$ in diameter) were deposited on the flat surfaces of the disk by means of magnetron sputtering. A custom sample holder with copper and brass contacts was used to measure both electrical and magnetic properties simultaneously. To measure the magnetic properties (complex ac susceptibility, dc magnetization) a home-built continuously reading SQUID magnetometer with immobile sample was used [9]. The magnetometer oper-

* corresponding author; e-mail: janu@fzu.cz ates at frequencies from dc up to $\approx 100 \mathrm{~Hz}$ and magnetic fields up to $20 \mathrm{mT}$ in non-persistent mode. dc electrical resistivity was measured using Agilent 34410 A digital multimeter (up to $10^{7} \Omega \mathrm{m}$, constant excitation current) and Keithley 6517 A electrometer (dc resistivity higher than $10^{6} \Omega \mathrm{m}$, constant excitation voltage). The Agilent 4284 A LCR meter was used to measure complex ac impedance at frequencies $20 \mathrm{~Hz}$ to $1 \mathrm{MHz}$. In this case the real and imaginary part of electrical current was measured ( $i_{1}$ and $i_{2}$, respectively) and the result was expressed in terms of AC resistivity $\rho=\left(u / i_{1}\right)(S / l)$ and relative permittivity $\varepsilon=(l / S) /\left(\omega \varepsilon_{0} u / i_{2}\right)$, where $u$ is the measuring voltage, $S$ is the sample surface, $l$ is the sample thickness, $\omega$ is the measuring angular frequency and $\varepsilon_{0}$ is the permittivity of vacuum.

\section{Results and discussion}

The dc resistivity of magnetite was measured in the temperature range from $15 \mathrm{~K}$ to $120 \mathrm{~K}$ (Fig. 1). Together with experimental data taken at different excitation currents (or voltages) and wide temperature range, a theoretical curve according to Mott's variable range hopping (VRH) model [10] shows very accurate fit. The parameters describing resistivity below $T_{\mathrm{V}}$ are $\rho_{0}=2.13 \times 10^{-13} \Omega \mathrm{m}$ and $T_{0}=1.04 \times 10^{8} \mathrm{~K}$. These parameters do not represent any physically meaningful quantities, so one should not get confused by the values themselves (temperature higher than that of the Sun core and resistivity lower than that of silver at room temperature).

Other similar measurements [11-13] suggest Arrhenius-like temperature-activated $\rho(T)$ dependence or Mott's VRH dependence valid only over limited temperature range.

Magnetic susceptibility of magnetite $\chi_{\mathrm{AC}}$ displays so-called glass-like transition [14] in the range of $30-50 \mathrm{~K}$. It manifests itself as a peak of the imaginary part of the complex ac susceptibility and a sigmoid-shaped transition of the real part, see Fig. 2b. The transition shifts towards higher temperatures for higher measuring frequencies $\omega$ and can be approximately described as a sim- 


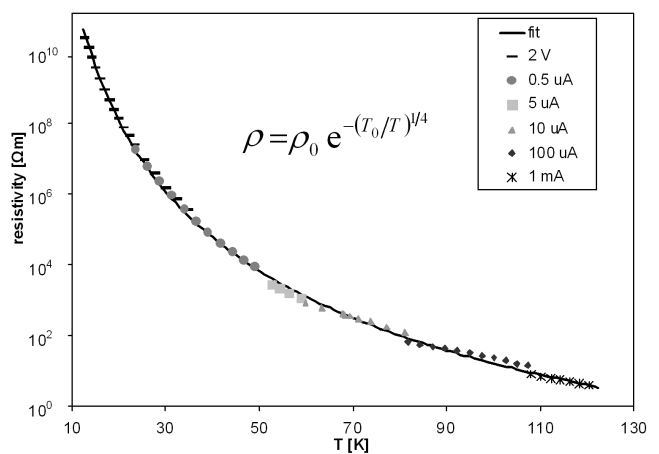

Fig. 1. The temperature dependence of the dc resistivity of magnetite sample measured both by an electrometer (at $2 \mathrm{~V}$ range) and common digital ohmmeter (ranges $0.5 \mu \mathrm{A}$ to $1 \mathrm{~mA}$ ) together with a fit (solid line) according to Mott's variable range hopping model.

ple Debye relaxation $\chi \approx 1 /(1-i \omega \tau)$ with temperature dependent relaxation time $\tau$.

In Fig. 2 this transition is compared to dielectric behavior in the same temperature range. Both real part of ac complex susceptibility and dielectric permittivity (Fig. 2a) display similar transitions at temperatures below $T_{\mathrm{V}}$ and both these transitions shift to higher temperatures with increasing measuring frequency according to the Arrhenius law $f=f_{0} \exp \left(-E_{0} / k_{\mathrm{B}} T\right)$ with parameters $f_{0}=7.88 \times 10^{9} \mathrm{~Hz}$ and $E_{0}=729 \mathrm{~K}$ for magnetic transition and $f_{0}=3.24 \times 10^{9} \mathrm{~Hz}$ and $E_{0}=839 \mathrm{~K}$ for dielectric transition.

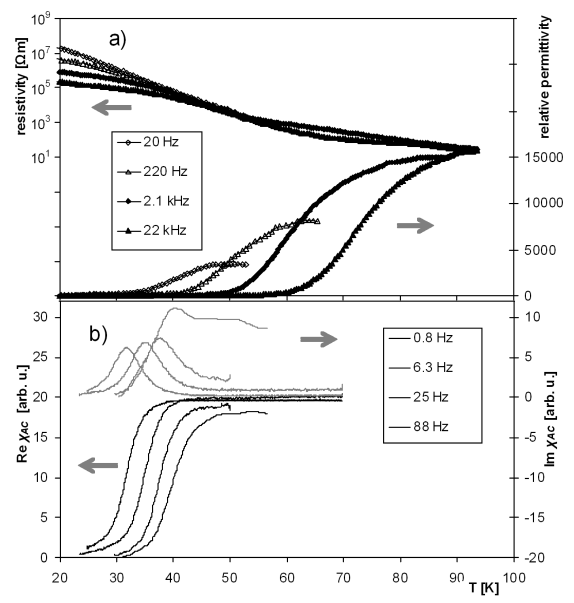

Fig. 2. The temperature dependence of (a) complex ac magnetic susceptibility and (b) AC dielectric properties (the resistivity $\rho$ and relative permittivity $\varepsilon$ ). The temperature axes are identical to allow direct comparison.

These findings alone do not allow us to draw a direct link between glass-like transition and dielectric transition below $T_{\mathrm{V}}$. There are some indications of interplay of electrical and magnetic effects apart from magnetoresistivity, namely changes of $T_{\mathrm{V}}$ by applied electric field [15] or changes of magnetic anisotropy by electric field [16]. It has also been established [17] that the magnetic sus- ceptibility (and the glass-like transition itself) can be diminished by applying strong dc magnetic field. This can be explained as being due to the reduction of the number of magnetic domains when a monocrystalline sample is cooled through $T_{\mathrm{V}}$ in sufficiently strong magnetic field (about $200 \mathrm{mT}$ ). Also the anomalously high relative permittivity in the order of $10^{4}$ can be explained as a result of some kind of domain structure where conducting domains and insulating domain walls (or grains and grain boundaries, respectively) can give rise to the MaxwellWagner relaxation [18].

Thus the idea of magnetic domains as a link between the magnetic glass-like transition and dielectric transition can be verified by measuring the dielectric properties of magnetite in strong dc magnetic field, a condition at which the magnetic transition disappears.

\section{Acknowledgments}

This work was supported by Institutional Research Plan Contract No. AVOZ10100520, Research Project MSM Contract No. 0021620834 and grant ESO MNT-ERA (ME10069 of MEYS CR) and SVV-2010-261303.

\section{References}

[1] E.J.W. Verwey, Nature (London) 144, 327 (1939).

[2] N.F. Mott, Rev. Mod. Phys. 40, 677 (1968).

[3] T. Toyoda, S. Sasaki, M. Tanaka, Am. Mineral. 84, 294 (1999).

[4] J.P. Wright, J.P. Attfield, P.G. Radaelli, Phys. Rev. Lett. 87, 266401 (2001).

[5] G. Subías, J. García, J. Blasco, M.G. Proietti, H. Renevier, M.C. Sánchez, Phys. Rev. Lett. 93, 156408 (2004).

[6] V.V. Shchennikov, S.V. Ovsyannikov, A.E. Karkin, S. Todo, Y. Uwatoko, Solid State Commun. 149, 759 (2009).

[7] Y.S. Dedkov, U. Rüdiger, G. Güntherodt, Phys. Rev. B 65, 64417 (2002).

[8] V.A.M. Brabers, J. Cryst. Growth 8, 26 (1971).

[9] Z. Janů, F. Soukup, R. Tichý, Int. J. Materials Product Technol. 26, 388 (2006).

[10] N.F. Mott, J. Non-Cryst. Solids 1, 1 (1968).

[11] N. Lenge, H. Kronmuller, F. Walz, J. Phys. Soc. Jpn. 53, 1406 (1984).

[12] M. Kobayashi, Y. Akishige, E. Sawaguchi, J. Phys. Soc. Jpn. 55, 4044 (1986).

[13] K. Mizushima, K. Nakao, S. Tanaka, J. Phys. Soc. Jpn. 44, 1831 (1978).

[14] Z. Janů, J. Hadač, Z. Švindrych, J. Magn. Magn. Mater. 310, e203 (2007).

[15] M. Ziese, H.J. Blythe, J. Phys., Condens. Matter 12, $13(2000)$.

[16] G.T. Rado, J.M. Ferrari, Phys. Rev. B 12, 5166 (1975).

[17] M. Bałanda, A. Wiecheć, D. Kim, Z. Kąkol, A. Kozłowski, P. Niedziela, J. Sabol, Z. Tarnawski, J.M. Honig, Eur. Phys. J. B 43, 201 (2005).

[18] J. Liu, C.G. Duan, W.G. Yin, W.N. Mei, R.W. Smith, J.R. Hardy, Phys. Rev. B 70, 144106 (2004). 